CLINICAL STUDY

\title{
Endothelial function, insulin sensitivity and inflammatory markers in hyperprolactinemic pre-menopausal women
}

\author{
Dilek Yavuz, Oğuzhan Deyneli, Ihsan Akpınar ${ }^{1}$, Erdem Yıldız ${ }^{1}$, Hülya Gözü, Özlem Sezgin, Goncagül Haklar ${ }^{2}$ \\ and Sema Akalın \\ Department of Internal Medicine, Section of Endocrinology and Metabolism, ${ }^{1}$ Department of Radiology and ${ }^{2}$ Department of Biochemistry, \\ Marmara University, Istanbul, Turkey
}

(Correspondence should be addressed to D Yavuz, Marmara Üniversitesi Hastanesi, Tophanelioglu cad no. 13-15, Altunizade, Üsküdar, Istanbul 34660, Turkey; Email: dyavuz@tnn.net)

\begin{abstract}
Background: Hyperprolactinemia has been reported to be associated with abnormalities of carbohydrate metabolism. The aim of this study was to evaluate the effects of hyperprolactinemia and bromocriptine $(\mathrm{Brc})$ treatment on endothelial function, insulin sensitivity and inflammatory markers in pre-menopausal women.

Methods: Sixteen hyperprolactinemic pre-menopausal women with pituitary adenomas were recruited and 20 healthy subjects were included as controls. Patients were given Brc in doses of $2.5-20 \mathrm{mg} / \mathrm{dl}$ until normal levels of prolactin were reached. Prior to treatment and 2 months after prolactin levels were normalized, the following tests were performed. Insulin sensitivity was determined by an oral glucose tolerance test based on a formula named the insulin sensitivity index (ISI composite). Endothelial function was measured as flow-mediated dilatation (FMD) on a brachial artery using high resolution ultrasound.

Results: Serum glucose, insulin, estrogen, highly sensitive C-reactive protein (hsCRP), fibrinogen, homocysteine and uric acid levels were measured. Calculated ISI composite and FMD were significantly lower in the hyperprolactinemic group in comparison with the controls and improved after Brc treatment. Serum homocysteine, hsCRP and uric acid levels were significantly higher in hyperprolactinemic patients than in the controls and returned to normal levels with Brc treatment. Serum prolactin concentrations were inversely correlated with FMD measurements $(r=-0.68 ; P<0.0001)$, ISI composite $(r=-0.48 ; P<0.005)$ and serum estrogen $(r=-0.54 ; P<0.005)$, and positively correlated with serum homocysteine concentrations $(r=0.55 ; P<0.0001)$ in the hyperprolactinemic group. Conclusions: The hyperprolactinemic state is associated with impaired endothelial function and decreased insulin sensitivity, which are early markers of atherosclerosis. These alterations may predispose to the development of atherosclerosis in non-treated cases. Correction of the hyperprolactinemic state is associated with improved endothelial function and insulin sensitivity.
\end{abstract}

European Journal of Endocrinology 149 187-193

\section{Introduction}

Although hyperprolactinemia is a common disorder, its exact metabolic consequences are unclear. Experiments in animals and humans showed that prolactin may exert a diabetogenic effect (1-3). Studies in hyperprolactinemic patients with or without pituitary tumors revealed hyperinsulinemia and reduced glucose tolerance $(3-7)$.

The impaired glucose metabolism in the hyperprolactinemic state may be explained by enhanced peripheral insulin resistance and/or altered pancreatic beta-cell function. Various experimental and clinical studies suggest that prolactin per se may induce some kind of insulin resistance. Decreased sensitivity of peripheral tissues to insulin has been documented $(4,8)$. Furthermore, islet beta cells treated with prolactin decrease the glucose stimulation threshold and enhance insulin secretion (9).
Although the strong association between insulin resistance and endothelial dysfunction is well recognized, the influence of hyperprolactinemia on the development of endothelial function has not been studied.

The aim of this study was to evaluate the effects of hyperprolactinemia and its treatment with bromocriptine (Brc) on endothelial function, and to examine the relationship between insulin resistance and inflammatory markers in hyperprolactinemic patients.

\section{Methods}

\section{Patients}

Sixteen hyperprolactinemic pre-menopausal women (group P) were recruited into the study following written informed consent. The protocol was approved by the local ethical committee of the Marmara University 
Hospital. Twenty healthy subjects matched for age and gender were included as controls (group C). The demographic characteristics of the study groups are presented in Table 1. A diagnosis of pituitary microadenoma was made either by an MRI scan or a computed axial tomographic scan in all hyperprolactinemic patients.

Formal tests of hypothalamic-pituitary function and pelvic ultrasonographic examination were normal. None of the patients had a concurrent illness other than hyperprolactinemia, and none of the participants were smokers or on any medication at the time of the study.

All hyperprolactinemic patients were studied prior to and following the suppression of circulating prolactin levels. Hyperprolactinemic patients were given Brc $(2.5-20 \mathrm{mg} / \mathrm{dl})$ and the dosage was titrated monthly until prolactin levels decreased below $20 \mathrm{ng} / \mathrm{dl}$, and the tests were repeated 2 months after reaching target prolactin levels. All measurements except the oral glucose tolerance test (OGTT) were repeated in the controls with a similar time delay. Controls and post-treatment hyperprolactinemic patients were studied in the early follicular phase.

\section{Insulin sensitivity}

Insulin sensitivity was determined by an OGTT based on the formula described by Matsuda and DeFronzo and named the insulin sensitivity index (ISI) composite (10). Whole-body insulin sensitivity during the OGTT was calculated by the following formula:

$$
\begin{aligned}
\text { ISI composite }= & {[10000 / \sqrt{ } \text { (fasting plasma glucose }} \\
& \times \text { fasting plasma insulin }) \\
& \times(\text { mean OGTT glucose concentration } \\
& \times \text { mean OGTT insulin concentration })]
\end{aligned}
$$

After an overnight fast, OGTTs (75 g glucose) were performed between 0800 and $0900 \mathrm{~h}$. Blood samples were taken just before ( $0 \mathrm{~min}$ ) and 30, 60, 90 and $120 \mathrm{~min}$ after the administration of glucose for the measurement of serum glucose and insulin concentrations.

The homeostatic model assessment $\left(\mathrm{HOMA}_{\mathrm{IR}}\right)$ was used (11). Area under the curves (AUC) of glucose and insulin during OGTT were calculated according to the trapezoid rule.

Table 1 Demographic characteristics of the study groups.

\begin{tabular}{lcc}
\hline & $\begin{array}{c}\text { Group P } \\
(n=16)\end{array}$ & $\begin{array}{c}\text { Group C } \\
(n=20)\end{array}$ \\
\hline Age (years) (mean士s.D.) & $31.1 \pm 9.7$ & $32.3 \pm 8.9$ \\
Amenorrhea (+/-) & $7 / 16$ & - \\
Galactorrhea (+/-) & $9 / 16$ & - \\
Hirsutism & $9 / 16$ & - \\
Duration of hyperprolactinemia (months) & $12 \pm 5$ & - \\
\hline
\end{tabular}

\section{Endothelial function}

Endothelial function was assessed with a non-invasive method described by Celermajer et al. (12). This noninvasive method evaluates endothelial function using post-ischemic (forearm) vasodilatation, which causes enhanced flow in the proximal brachial artery and consequently a shear stress-induced vasodilatation which is regarded as endothelium dependent.

Endothelium-dependent vasodilatation was measured using a high resolution ultrasound (GE Logic 700, Horten, Norway) with a $8.5 \mathrm{MHz}$ linear-array transducer. Subjects had to rest for at least $10 \mathrm{~min}$ before the first scan was recorded. Increased flow was induced by deflating a pneumatic tourniquet after a 5-min suprasystolic arterial forearm compression. The post-ischemic scan was performed 45-60 s after cuff deflation. To test endothelium-independent dilatation, further scans were performed at rest and $4 \mathrm{~min}$ after sublingual administration of $0.4 \mathrm{mg}$ glyceryltrinitrate (GTN) as a direct nitric oxide donor. The time interval between the first and second measurements was $20 \mathrm{~min}$ for vessel recovery.

Vessel diameters were analyzed on frozen images over the length of an artery of $>1 \mathrm{~cm}$. The difference in lumen diameter between rest and reactive hyperemia, expressed as percent change was regarded as endothelium-dependent (\% flow-mediated dilatation (\%FMD)), and GTN\% as endothelium-independent vasodilatation.

\section{Assays}

Serum prolactin levels were measured by an electroluminescence assay (Roche Elecsys 2010, Roche Diagnostics $\mathrm{GmbH}$, Mannheim, Germany). The interand intra-assay coefficients of variations were $2.8 \%$ and $3.4 \%$ respectively for a measurement range from 72 to $2332 \mathrm{mg} / \mathrm{dl}$. Insulin concentrations were quantified with an Immulite analyzer using an immunometric method (DPC, Los Angeles, CA, USA).The intra-assay precision ranged from 4.8 to $5.4 \%$ and total precision ranged from 4.8 to $7.6 \%$ over a mean range from 10 to $439 \mathrm{mU} / \mathrm{ml}$. Serum estrogen levels were measured by an electrochemiluminescence assay with the intra-assay variation ranging from 6.3 to $15 \%$ over a concentration range from 46 to $480 \mathrm{pg} / \mathrm{ml}$. The inter-assay variation ranged from 6.4 to $16 \%$ over a concentration range from 56 to $486 \mathrm{pg} / \mathrm{ml}$. Analytical sensitivity was $15 \mathrm{pg} / \mathrm{ml}$. Glucose was determined by an enzymatic colorimetric assay. Fibrinogen levels were measured in plasma by the clotting method with an excess of thrombin (STA, Diagnostica Stago, Asnieres-Sur-Siene, France). The intra-assay variation ranged from 1.4 to $3.9 \%$ over a concentration range from 139 to $283 \mathrm{mg} / \mathrm{dl}$, and the interassay variation from 2.1 to $3.6 \%$ over a concentration range from 136 to $275 \mathrm{mg} / \mathrm{dl}$. 
Homocysteine levels were measured with a fluorescence polarization immunoassay technology (IMX, Abbott, Wiesbaden, Germany). The intra-assay variation ranged from 1.4 to $2.2 \%$ and total precision from 3.7 to $5.2 \%$ over a concentration range from 5.9 to $21.6 \mu \mathrm{mol} / \mathrm{l}$.

Highly sensitive C-reactive protein (hsCRP) levels were measured by an immunoturbidimetric assay (Roche Diagnostics $\mathrm{GmbH}$ ). The within-run coefficient of variation ranged from 0.6 to $1.3 \%$ over a concentration range from 2.3 to $9.4 \mathrm{mg} / \mathrm{dl}$, while the day-today coefficient of variation ranged from 1.3 to $6.0 \%$ for concentrations between 2.2 and $11.5 \mathrm{mg} / \mathrm{dl}$. The lower detection limit was $0.3 \mathrm{mg} / \mathrm{dl}$.

Serum total cholesterol, uric acid and triglyceride levels were measured by enzymatic colorimetric assays (Roche Diagnostics $\mathrm{GmbH}$ ). The within-run and between-day coefficients of variation were $0.8 \%$ and $1.7 \%$ for total cholesterol, $0.5 \%$ and $1.7 \%$ for uric acid and $1.5 \%$ and $1.8 \%$ for triglyceride assays.

Serum low-density lipoprotein (LDL) cholesterol levels were determined by a direct automated method using polyethylene glycol-modified enzymes, sulfated cyclodextrin and dextran sulfate (Roche Diagnostics $\mathrm{GmbH})$. The within-run and between-day coefficients of variation were $1.3 \%$ and $2.6 \%$ for a mean high-density lipoprotein (HDL) cholesterol level of $23 \pm 0.6 \mathrm{mg} / \mathrm{dl}$.

Plasma glucose levels were analyzed with the glucoxidase method. The within-run coefficient of variation was $0.9 \%$ for a mean concentration of $116 \mathrm{mg} / \mathrm{dl}$ and the between-day variation $1.8 \%$ for a mean concentration of $123 \mathrm{mg} / \mathrm{dl}$.

\section{Statistical analysis}

All calculations and statistical analysis were performed with the Statistical Package for Social Sciences (SPSS Inc., Chicago, IL, USA). Comparisons between the groups were performed using the paired $t$-test and Student's $t$-test where appropriate. Correlation analyses were performed with linear regression. AUC was calculated according to the trapezoid rule. The level of statistical significance was set at $P<0.05$. The results are expressed as means \pm S.D. Stepwise multiple regression analysis was used in multivariate analysis with endothelial dysfunction or insulin sensitivity index as the dependent variable.

\section{Results}

No significant differences were observed in systolic and diastolic blood pressure measurements or body mass index (BMI) values between the two groups (Table 2). All hyperprolactinemic patients reached normal prolactin levels $(<20 \mathrm{mg} / \mathrm{dl})$ within $2-4$ months after starting Brc. Fourteen out of 16 patients $(87.5 \%)$ complained of nausea, dizziness or sleep disturbances but none discontinued the treatment.

Serum prolactin levels were naturally lower in healthy controls than in the hyperprolactinemic patients. Serum estrogen levels were significantly lower in non-treated hyperprolactinemic subjects compared with the controls and post-treatment levels. Serum homocysteine and hsCRP levels were significantly higher in hyperprolactinemic patients in comparison with the healthy controls and Brc-treated patients. There were no differences in serum cholesterol, triglyceride, HDL cholesterol and LDL cholesterol concentrations between the groups (Table 3 ).

Healthy subjects had higher ISI composite values than before treatment hyperprolactinemic patients, while post-treatment values were similar to that of controls (Table 4). HOMA IR were significantly decreased following Brc treatment in hyperprolactinemic patients $(P<0.01)$. AUC for glucose and insulin during OGTT were significantly decreased following Brc treatment in hyperprolactinemic patients $(P<0.01$ and $P<0.05$ for glucose and insulin respectively). AUC for glucose and insulin were similar in controls and in the patients with hyperprolactinemia after treatment. Basal glucose and insulin levels were not different between the groups. Serum glucose and insulin levels during OGTT are shown in Fig. 1. Endothelial function measured as FMD was significantly improved after Brc therapy $(6.65 \pm 3.0 \%$ and $12.9 \pm 3.1 \%$ before and after Brc treatment respectively) in hyperprolactinemic patients $(P<0.0001)$. Healthy subjects $(11.7 \pm 3.9 \%$ and $12.0 \pm 4.2 \%$ baseline and final measurements respectively) and Brc-treated hyperprolactinemic patients had similar FMD values. GTN values were not different

Table 2 Blood pressure measurements and BMI in the study groups on two occasions. Values are means \pm S.D.

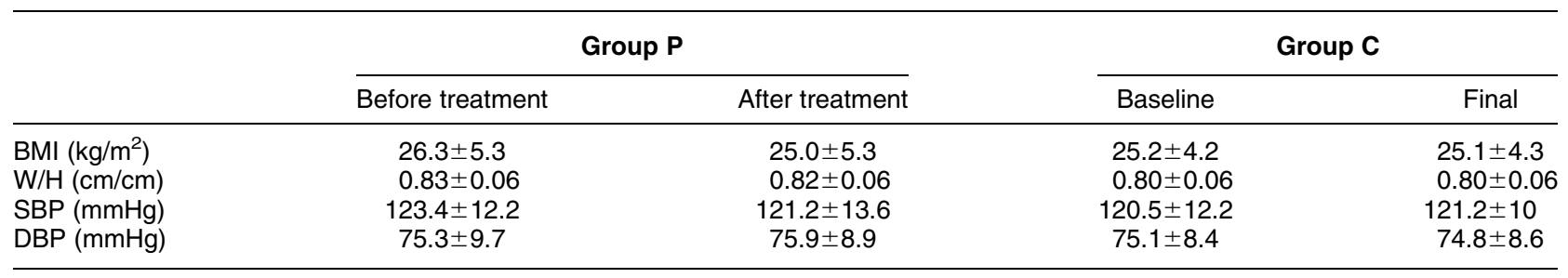

W/H, Waist/Hip; SBP, systolic blood pressure; DBP, diastolic blood pressure. 
Table 3 Serum concentrations of prolactin, estrogen, lipids and inflammation markers in the study groups on two occasions. Values are means \pm S.D.

\begin{tabular}{|c|c|c|c|c|}
\hline & \multicolumn{2}{|c|}{ Group P } & \multicolumn{2}{|c|}{ Group C } \\
\hline & Before treatment & After treatment & Baseline & Final \\
\hline Prolactin (ng/dl) & $156.5 \pm 77.2^{*}$ & $6.0 \pm 5.8$ & $14.9 \pm 4.9$ & $15.3 \pm 4.2$ \\
\hline Estrogen (ng/ml) & $50.1 \pm 19.2 \dagger$ & $129.9 \pm 60.8$ & $124.8 \pm 56.7$ & $130.7 \pm 56.8$ \\
\hline Homocysteine $(\mu \mathrm{mol} / \mathrm{l})$ & $12.4 \pm 3.3 \ddagger$ & $8.6 \pm 2.0$ & $8.6 \pm 1.6$ & $9.0 \pm 1.8$ \\
\hline Fibrinogen $(\mathrm{mg} / \mathrm{dl})$ & $3.05 \pm 0.4$ & $3.03 \pm 0.4$ & $3.09 \pm 0.7$ & $3.2 \pm 0.7$ \\
\hline $\mathrm{hsCRP}(\mathrm{mg} / \mathrm{dl})$ & $3.51 \pm 0.5 \S$ & $2.46 \pm 1.3$ & $2.96 \pm 0.6$ & $2.99 \pm 0.6$ \\
\hline Uric acid (mg/dl) & $3.9 \pm 1.09$ & $3.3 \pm 1.0$ & $3.4 \pm 1.0$ & $3.5 \pm 0.9$ \\
\hline Total cholesterol $(\mathrm{mg} / \mathrm{dl})$ & $195.2 \pm 31$ & $184.6 \pm 38$ & $179.7 \pm 44$ & $178.1 \pm 84$ \\
\hline Triglycerides (mg/dl) & $83.7 \pm 28$ & $78 \pm 25$ & $87.8 \pm 33$ & $85.9 \pm 32$ \\
\hline $\mathrm{HDL}(\mathrm{mg} / \mathrm{dl})$ & $55.6 \pm 12$ & $49.6 \pm 10$ & $49.2 \pm 11$ & $48.8 \pm 11$ \\
\hline $\mathrm{LDL}(\mathrm{mg} / \mathrm{dl})$ & $123.5 \pm 30$ & $117.3 \pm 35$ & $113.9 \pm 37$ & $112.8 \pm 36$ \\
\hline
\end{tabular}

${ }^{*} P<0.001$ vs after treatment and group $\mathrm{C}$.

$+P<0.0001$ vs after treatment and group $\mathrm{C}$.

$\ddagger P<0.01$ vs after treatment and group $\mathrm{C}$.

$\S P<0.05$ vs after treatment, $P<0.001$ vs group $C$.

१ $P<0.0001$ vs after treatment.

Table 4 Insulin sensitivity indices, serum glucose and insulin levels and AUC calculations during OGTT in the patient groups before and after treatment with Brc and in the control group. Values are means \pm S.D.

\begin{tabular}{lccc}
\hline & \multicolumn{3}{c}{ Group P } \\
\cline { 2 - 3 } & Before treatment & After treatment & Group C \\
\hline ISI composite & $1.234 \pm 0.5$ & $1.816 \pm 0.5^{\star}$ & $2.02 \pm 0.6 \dagger$ \\
HOMA & $1.521 \pm 0.42 \S$ & $1.57 \pm 0.48 \ddagger$ \\
Glucose $(\mathrm{mg} / \mathrm{dl})$ & $2.127 \pm 1.1$ & $75.8 \pm 8.7$ & $77.1 \pm 8.4$ \\
Insulin $(\mathrm{mU} / \mathrm{ml})$ & $79.0 \pm 9.7$ & $8.15 \pm 2.7$ & $8.82 \pm 3$ \\
Glucose AUC $(\mathrm{mg} \mathrm{h} / \mathrm{ml})$ & $10.9 \pm 5.1$ & $64.54 \pm 519$ & $64.3 \pm 50 \uparrow$ \\
Insulin AUC $(\mathrm{mU} \mathrm{h} / \mathrm{ml})$ & $107.03 \pm 58$ & $60.53 \pm 23.9^{\star \star}$ & $55.1 \pm 32 \dagger \dagger$ \\
\hline
\end{tabular}

* $P<0.05$ vs before treatment.

$\dagger P<0.01$ vs before treatment.

$\ddagger P<0.05$ vs before treatment.

$\S P<0.01$ vs before treatment.

१ $P<0.01$ vs before treatment.

** $P<0.05$ vs before treatment.

$\dagger+P<0.01$ vs before treatment.

between the groups at baseline or at final evaluation. There was no difference in endothelium-independent dilatation between the groups $(17.3 \pm 3.2 \%$, $19.4 \pm 4.1 \%$, and $18.2 \pm 2.6 \%, 18.9 \pm 3.1 \%$ for baseline and final measurements in group $\mathrm{P}$ and group $\mathrm{C}$ respectively) (Fig. 2). Serum prolactin concentrations were inversely related to FMD $(r=-0.68 ; P<0.0001)$, ISI composite $(r=-0.48 ; P<0.005)$ and serum estrogen $(r=-0.54 ; P<0.005)$, while there was a direct correlation between serum prolactin levels and serum homocysteine concentrations $(r=0.62 ; P<0.0001)$ (Fig. 3) and with $\mathrm{HOMA}_{\mathrm{IR}}(r=0.60 ; P<0.0001)$ in the hyperprolactinemic group.

There were direct correlations between FMD and ISI composite $(r=0.39 ; P<0.05)$, and serum estrogen levels ( $r=0.56$; $P<0.0001)$, whereas an inverse correlation was observed with serum homocysteine concentrations $(r=-0.43 ; P<0.005)$. A significant positive correlation was observed between serum estrogen levels and ISI composite $(r=0.41 ; P<0.0001)$ in all study groups.

The duration of hyperprolactinemia or Brc dosage did not affect the endothelial function.

Stepwise multiple regression analysis of all univariate significant variables including variables that failed to reach significance (age, lipids) with FMD as the dependent variable, showed serum prolactin $(P<0.005)$ and estrogen concentrations $(P<0.005)$ to be independent risk factors for endothelial dysfunction. ISI composite as an independent variable was not found to be significantly associated with any variable.

\section{Discussion}

Hyperprolactinemia is a common disorder with poorly defined metabolic consequences. The present data demonstrates that the hyperprolactinemic state is associated with glucose intolerance. Our results are in 


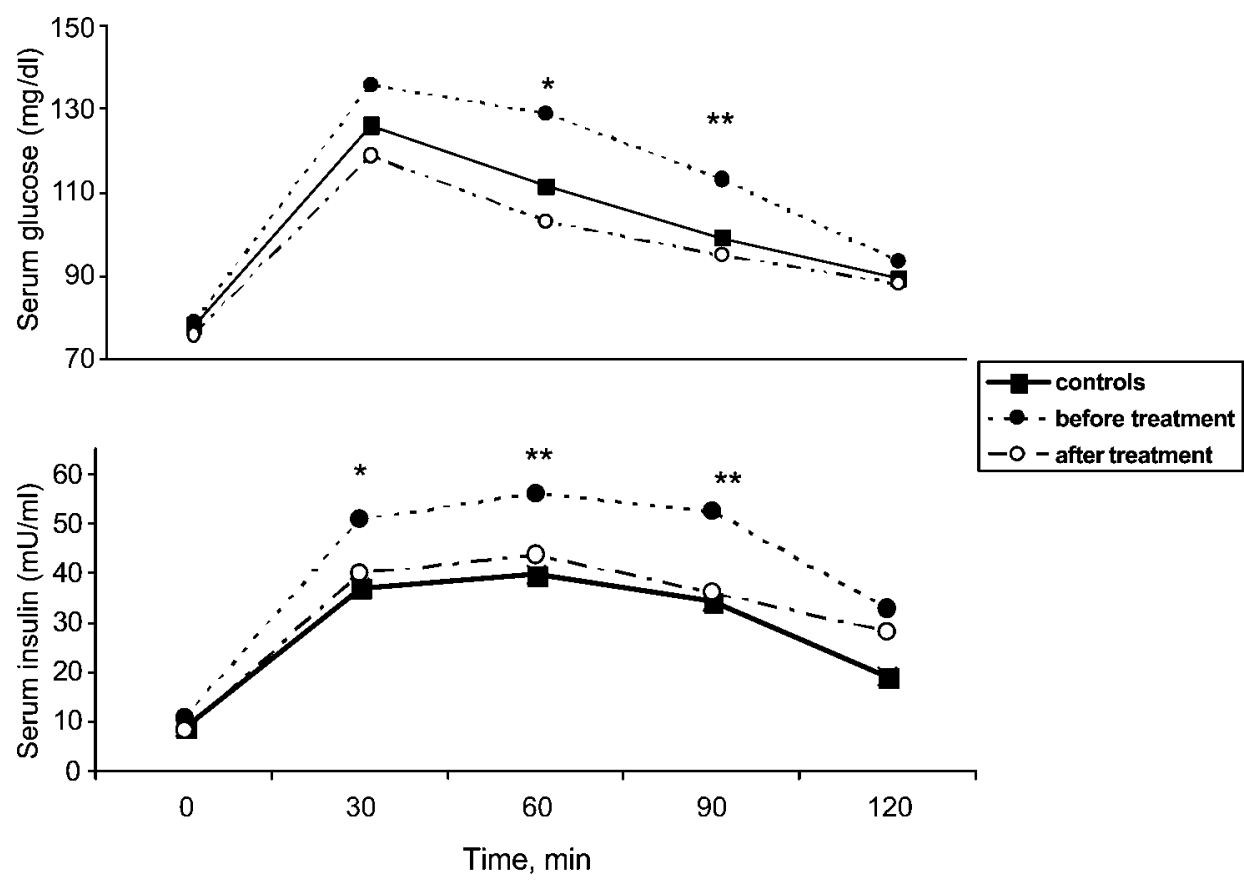

Figure 1 Glucose and insulin levels during OGTT in the patient group before and after treatment with Brc and in the control group. Glucose concentrations at 60 and $90 \mathrm{~min}$ and insulin concentrations at 30,60 and $90 \mathrm{~min}$ are significantly higher in the hyperprolactinemic patients than in the healthy controls. ${ }^{\star} P<0.05$, ${ }^{\star \star} P<0.01$ vs control group.

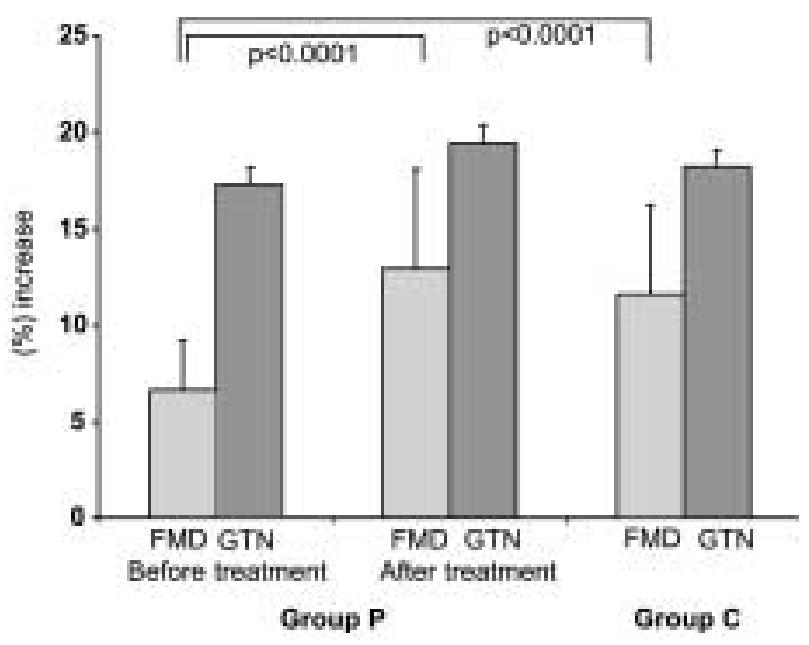

Figure 2 FMD measurements in the patient group before and after treatment with Brc and in the control group. FMD was lower in the hyperprolactinemic patients than in the controls

$(P<0.0001)$ and increased significantly after Brc treatment $(P<0.0001)$. FMD: endothelium-dependent dilatation, GTN: endothelium-independent dilatation.

agreement with the findings of Landgraf et al. who showed that hyperprolactinemic patients had decreased glucose tolerance and hyperinsulinemia following a glucose load and suggested that prolactin is a diabetogenic hormone (3).

Although the gold standard of measuring insulin action is the euglycemic clamp technique (13), the ISI composite has been validated as a simple and reasonable estimate of the whole-body insulin sensitivity and has been applied in this study (10). The altered glucose tolerance observed in our patients may be due to reduced insulin sensitivity, because the ISI composite was significantly lower in patients in comparison with the controls.

The results of this study might indicate a causal relationship between hyperprolactinemia and the combination of altered glucose tolerance and reduced insulin sensitivity. A significant relationship between prolactin and insulin sensitivity indexes ISI composite and $\mathrm{HOMA}_{\mathrm{IR}}$ might indicate a direct effect of prolactin on insulin sensitivity.

Hyperinsulinemia observed in hyperprolactinemic patients during OGTT may be due to direct beta-cytotrophic action of prolactin on the islets. In vivo and in vitro studies indicate that prolactin alters insulin secretory characteristics $(14,15)$ and leads to hyperinsulinemia that resembles insulin resistance $(3-5,8)$.

Factors known to influence insulin sensitivity (age, body weight and fasting glucose) were similar in patients and controls. The BMI change observed among the patients during Brc treatment may have a clinical impact, although it was not statistically significant. The non-significant weight loss could be due to gastrointestinal side effects of Brc.

Restored gonadal function during therapy could play a central role in determining endothelial function and insulin sensitivity in hyperprolactinemic pre-menopausal women. Increased estrogen levels may have an 

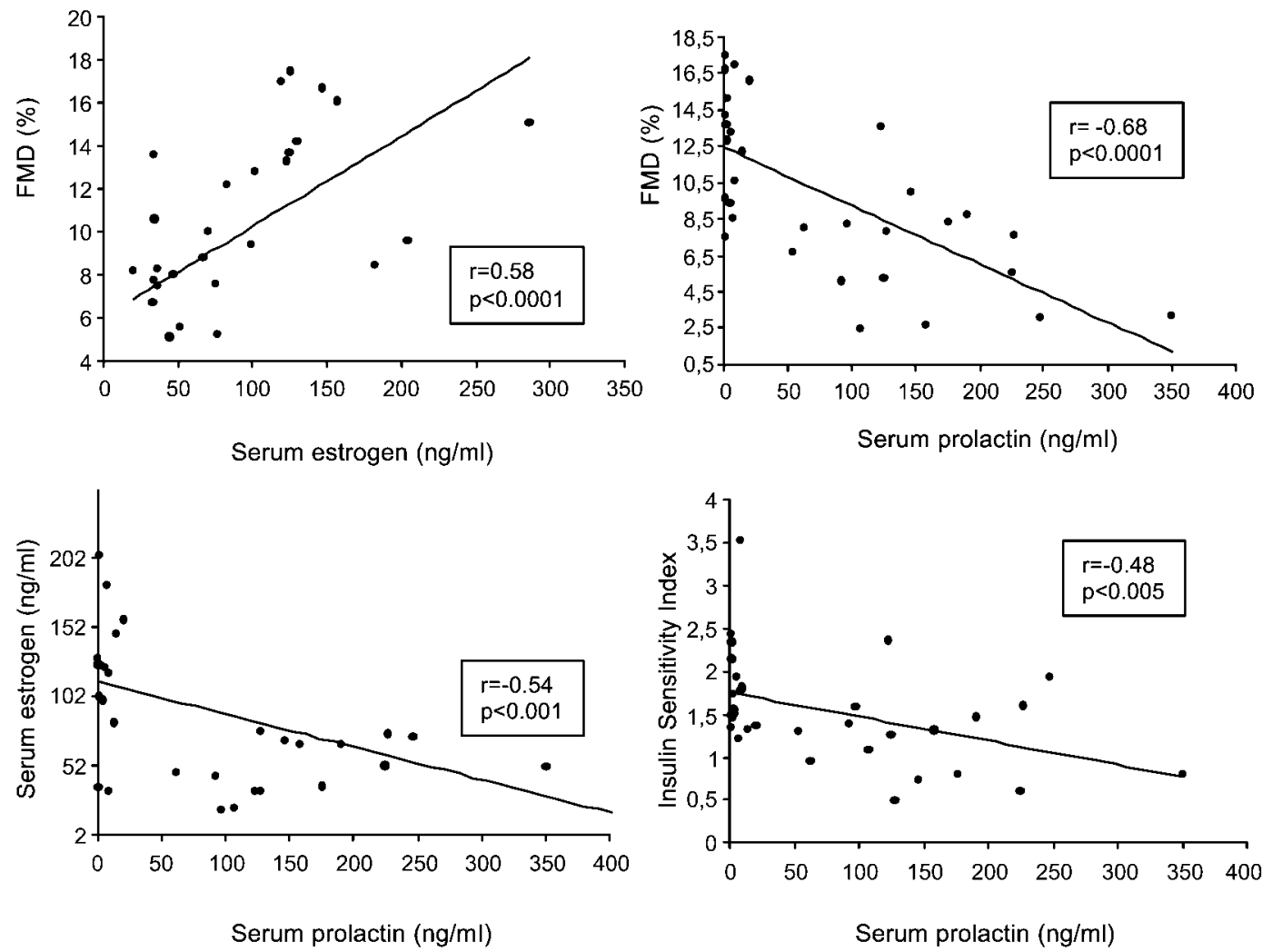

Figure 3 Correlation analysis between prolactin concentrations and estrogen levels, FMD and ISI composite in the subjects studied. Prolactin concentrations were inversely correlated with estrogen levels, FMD and ISI composite. There was a positive correlation between estrogen concentrations and FMD among the patients.

additive effect on metabolic and vascular parameters. As the coexistence of polycystic ovaries were excluded, insulin resistance seems to be associated with the hyperprolactinemic state in our hyperprolactinemic patients.

Direct effects of Brc treatment on insulin sensitivity could not be excluded in this study, and the data about the effects of Brc on glucose metabolism are conflicting. Furthermore, the effects of Brc on endothelial function are unknown. While some studies indicate that Brc improves glycemic control and glucose tolerance in obese patients with type 2 diabetes (16), others reported no effect of Brc on glucose metabolism (3). Further studies are needed to clarify the direct effects of Brc on insulin sensitivity and endothelial function in hyperprolactinemic patients.

Endothelial dysfunction in hyperprolactinemic patients was another prominent finding in this study. Endothelial function measured as FMD was lower in hyperprolactinemic patients in comparison with the healthy subjects and improved during Brc treatment. Endothelial dysfunction could be due to hyperprolactinemia, low estrogen concentrations or insulin resistance in hyperprolactinemic patients. The stepwise regression analysis indicated that serum prolactin and estrogen levels are independent risk factors for endothelial dysfunction in hyperprolactinemic patients.
Reports regarding the association between FMD and estrogen levels are conflicting. Mather et al. found no such association (17), whilst Higashi et al. reported improved endothelial function following estrogen replacement in post-menopausal women (18).

Endothelial cell dysfunction is proposed to be an early event of atherogenesis $(19,20)$. Previous studies reported a relationship between indirect markers of endothelial cell activation and inflammatory markers $(21,22)$. Low-grade chronic inflammation, characterized by elevated concentrations of hsCRP, is associated with increased risk of atherosclerotic cardiovascular disease (21-23) and endothelial dysfunction (23). In the present study, hsCRP levels were significantly higher in hyperprolactinemic patients than in the healthy subjects and decreased during treatment. There was an inverse correlation between serum CRP levels and FMD in all study groups. These results indicate that low-grade inflammation accompanies hyperprolactinemia and endothelial dysfunction.

Hyperhomocysteinemia is a non-traditional marker of atherosclerosis and is known to be associated with alterations of endothelial and smooth muscle cell function $(24,25)$. Previous data indicate that homocysteine levels are associated with hyperinsulinemia $(26,27)$ and are independently associated with insulin resistance (28). Homocysteine concentrations were higher in 
hyperprolactinemic patients than in healthy controls, decreased during Brc treatment and correlated with the serum prolactin levels.

In conclusion, hyperprolactinemia is associated with insulin resistance, endothelial dysfunction and low grade inflammation, all of which were reverted by Brc treatment. As these parameters are determinants of the atherosclerotic process, hyperprolactinemia may be a factor predisposing to atherosclerosis. This is the first report of a case-controlled analysis indicating that hyperprolactinemia may be associated with endothelial dysfunction and low grade inflammation. Further studies are required in order to clarify whether hyperprolactinemic patients are at an increased risk of cardiovascular morbidity and mortality.

\section{Acknowledgement}

The authors wish to thank to Miss Aynur Altun for her technical assistance.

\section{References}

1 Reis FM, Reis AM \& Coimbra CC. Effects of hyperprolactinemia on glucose tolerance and insulin release in male and female rats. Journal of Endocrinology 1977153 423-428.

2 Matsuda M \& Mori T. Effect of estrogen on hyperprolactinemiainduced glucose intolerance in SHN mice. Proceedings of the Society for Experimental Biology and Medicine 1996212 $243-247$.

3 Landgraf R, Landraf-Leurs MM, Weissmann A, Horl R, von Werder K \& Scriba PC. Prolactin: a diabetogenic hormone. Diabetologia 197713 99-104.

4 Serri O, Beauregard H, Rasio E \& Hardy J. Decreased sensitivity to insulin in women with microprolactinomas. Fertility and Sterility $198645572-574$.

5 Seki K \& Nagata I. Levels of glucose and insulin during twentyfour hours in hyperprolactinemic women with pituitary microadenoma. Gynecology and Obstetrics Investigations 199131 $222-225$.

6 Gustafson AB, Banasiak MF, Kalkhoff RK, Hagen TC \& Kim HJ. Correlation of hyperprolactinemia with altered plasma insulin and glucagon: similarity to effects of late human pregnancy. Journal of Clinical Endocrinology and Metabolism $1980 \mathbf{5 1}$ 242-246.

7 Foss MC, Paula FJ, Paccola GM \& Piccinato CE. Peripheral glucose metabolism in human hyperprolactinemia. Clinical Endocrinology $199543721-726$.

8 Schernthaner G, Prager R, Punzengruber C \& Luger A. Severe hyperprolactinemia is associated with decreased insulin binding in vitro and insulin resistance in vivo. Diabetologia $1985 \mathbf{2 8}$ $138-142$.

9 Sorenson RL, Brelje TC, Hegre OD, Marshall S, Anaya P \& Sheridan JD. Prolactin (in vitro) decreases the glucose stimulation threshold, enhances insulin secretion and increases dye coupling among islet beta cells. Endocrinology $1987 \mathbf{1 2 1}$ $1447-1453$

10 Matsuda M \& DeFronzo RA. Insulin sensitivity indices obtained from oral glucose tolerance testing. Diabetes Care 199922 $1462-1470$.

11 Matthews DR, Hosker JP, Rudenski AS, Naylor BA, Treacher DF \& Turner RC. Homeostasis model assessment: insulin resistance and beta cell function from fasting plasma glucose and insulin concentrations in man. Diabetologia 198528 412-419.

12 Celermajer DS, Sorensen KE, Gooch VM, Spiegelhalter DJ, Miller OI, Sullivan ID et al. Non-invasive detection of endothelial dysfunction in children and adults at risk of atherosclerosis. Lancet 1992340 1111-1115.

13 Ferrannini E \& Mari A. How to measure insulin sensitivity. Journal of Hypertension 199816 895-906.

14 Nielsen JH. Effects of growth hormone, prolactin and placental lactogen on insulin content and release and deoxyribonucleic acid synthesis in cultured pancreatic islets. Endocrinology 1982 $110600-608$.

15 Hoiriis Nielsen J, Nielsen V, Molsted Pedersen L \& Deckert T. Effects of pregnancy hormones on pancreatic islets in organ culture. Acta Endocrinologica $1986111336-341$.

16 Pijl H, Ohashi S, Matsuda M, Miyazaki Y, Mahankali A, Kumar V et al. Bromocriptine: a novel approach to the treatment of type 2 diabetes. Diabetes Care 200023 1154-1161.

17 Mather KJ, Verma S, Corenblum B \& Anderson TJ. Normal endothelial function despite insulin resistance in healthy women with the polycystic ovary syndrome. Journal of Clinical Endocrinology and Metabolism 200085 1851-1856.

18 Higashi Y, Sanada M, Sasaki S, Nakagawa K, Goto C, Matsuura $\mathrm{H}$ et al. Effect of estrogen replacement therapy on endothelial function in peripheral resistance arteries in normotensive and hypertensive postmenopausal women. Hypertension 20017 651-657.

19 Zeiher AM, Drexler H, Wollschlager H \& Just H. Endothelial dysfunction of the coronary microvasculature is associated with coronary blood flow regulation in patients with early atherosclerosis. Circulation 199184 1984-1992.

20 Deedwania PC. Endothelium: a new target for cardiovascular therapeutics. Journal of the American College of Cardiology 2000 $3567-70$.

21 Ross R. Atherosclerosis an inflammatory disease. New England Journal of Medicine 1999340 115-126.

22 Cleland SJ, Sattar N, Petrie JR, Forouhi NG, Elliott HL \& Connell JM. Endothelial dysfunction as a possible link between C-reactive protein levels and cardiovascular disease. Clinical Science 200098 531-535.

23 Fichtlscherer S, Rosenberger G, Walter DH, Breuer S, Dimmeler S \& Zeiher AM. Elevated C-reactive protein levels and impaired endothelial vasoreactivity in patients with coronary artery disease. Circulation 2000102 1000-1006.

24 Herrmann W. The importance of hyperhomocysteinemia as a risk factor for diseases: an overview. Clinical Chemistry and Laboratory Medicine 200139 666-674.

25 Fonseca V, Guba SC \& Fink LM. Hyperhomocysteinemia and the endocrine system, implications for atherosclerosis and thrombosis. Endocrine Reviews 199920 738-759.

26 Meigs JB, Jacques PF, Selhub J, Singer DE, Nathan DM, Rifai N et al. Fasting plasma homocysteine levels in the insulin resistance syndrome: the Framingham Offspring Study. Diabetes Care 2001 24 1403-1410.

27 Bar-On H, Kidron M, Friedlander Y, Ben-Yehuda A, Selhub J, Rosenberg IH et al. Plasma total homocysteine levels in subjects with hyperinsulinemia. Journal of Internal Medicine $2000 \mathbf{2 4 7}$ 287-294.

28 De Pergola G, Pannacciulli N, Zamboni M, Minenna A, Brocco G, Sciaraffia M et al. Homocysteine plasma levels are independently associated with insulin resistance in normal weight, overweight and obese premenopausal women. Diabetes, Nutrition and Metabolism $2001 \mathbf{1 4} 253-258$.

Received 17 December 2002

Accepted 4 June 2003 